\title{
Evaluation of treatment-related mortality among paediatric cancer deaths: a population based analysis
}

\author{
Jason D Pole ${ }^{1}$, Paul Gibson ${ }^{2}$, Marie-Chantal Ethier ${ }^{3}$, Tanya Lazor $^{3}$, Donna L Johnston ${ }^{4}$, Carol Portwine ${ }^{5}$,
} Mariana Silva ${ }^{6}$, Sarah Alexander ${ }^{7}$ and Lillian Sung ${ }^{\star 3,7}$ on behalf of the International Pediatric Oncology Mortality Classification (IPOMC) Group

${ }^{1}$ Pediatric Oncology Group of Ontario, 480 University Ave., Toronto, Ontario, M5G 1V2, Canada; ${ }^{2}$ Division of Haematology/ Oncology, Children's Hospital, London Health Sciences Centre, 800 Commissioners Rd. E., London, Ontario, N6A 5W9, Canada; ${ }^{3}$ Program in Child Health Evaluative Sciences, The Hospital for Sick Children, Peter Gilgan Centre for Research and Learning, 686 Bay St., Toronto, Ontario, M5G 0A4, Canada; ${ }^{4}$ Department of Pediatrics, Division of Hematology/Oncology, Children's Hospital of Eastern Ontario, 401 Smyth Rd, Ottawa, Ontario, K1H 8L1, Canada; ${ }^{5}$ Department of Pediatrics, McMaster Children's Hospital, 1280 Main St West, Hamilton, Ontario, L8N 3Z5, Canada; ${ }^{6}$ Department of Pediatrics, Kingston General Hospital, 76 Stuart St, Kingston, Ontario, K7L 2V7, Canada and 'Department of Pediatrics, Division of Haematology/Oncology, The Hospital for Sick Children, 555 University Ave., Toronto, Ontario, M5G 1X8, Canada

Background: Objectives were to describe the proportion of deaths due to treatment-related mortality (TRM) and to identify risk factors and probable causes of TRM among paediatric cancer deaths in a population-based cohort.

Methods: We included children with cancer $\leqslant 18$ years diagnosed and treated in Ontario who died between January 2003 and December 2012. Deaths were identified using a provincial registry, the Pediatric Oncology Group of Ontario Networked Information System. Probable causes of TRM were described.

Results: Among the 964 deaths identified, 821 were included. The median age at diagnosis was 6.6 years (range 0-18.8) and 51.8\% had at least one relapse. Of the deaths examined, TRM occurred in 217/821 (26.4\%) while 604/821 (73.6\%) were due to progressive cancer. Deaths from TRM did not change over time. Using multiple regression, younger age, leukaemia diagnosis and absence of relapse were independently positively associated with TRM. The most common probable causes of TRM were respiratory, infection and haemorrhage.

Conclusions: TRM was responsible for $26.4 \%$ of deaths in paediatric cancer. Underlying diagnosis, younger age and absence of relapse were associated with TRM and causes of TRM differed by diagnosis group. Future work should evaluate TRM rate and risk factors among newly diagnosed cancer patients.

Most children diagnosed with cancer in Canada, the United States and Europe will be cured (Craft, 2000). As the success of cancerdirected strategies continues to improve, treatment-related mortality (TRM), a devastating outcome of a cancer diagnosis, is becoming increasingly important (Ethier et al, 2011; Blanco et al, 2012). In order to reduce TRM, it is first important to understand its frequency and associated risk factors. Understanding TRM versus cancer-related death is fundamental to identifying the best strategies to improve survival. If progressive cancer is the primary cause of death, then strategies will need to focus on treatment intensification or innovative

*Correspondence: Dr L Sung; E-mail: lillian.sung@sickkids.ca

Received 26 October 2016; revised 2 December 2016; accepted 8 December 2016; published online 17 January 2017

(c) 2017 Cancer Research UK. All rights reserved 0007-0920/17 
approaches to cancer control. However, if TRM is the primary cause of death, then strategies will need to focus on enhancing supportive care or may even consider a reduction in treatment intensity.

We previously identified the lack of consistency in TRM definitions, which made epidemiological studies and comparisons between trials difficult or impossible to interpret (Ethier et al, 2011; Tran et al, 2014). For patients with TRM, we also noted the lack of a consistent cause-of-death attribution system, for example, criteria to define infection or haemorrhage as the cause of death. Consequently, we recently developed a standardised TRM definition and cause-of-death attribution system (Alexander et al, 2015). With this system, TRM is defined by death occurring in the absence of progressive cancer. This approach is important as many children with refractory disease will die from toxicities of therapy. The system allows flexibility as individual trials may choose to censor patients who relapse or those who undergo haematopoietic stem cell transplantation (HSCT). The developed TRM system was reliable and demonstrated criterion validity (Alexander et al, 2015).

Little is known about the epidemiology of TRM using this novel definition and cause-of-death attribution system. Taking a population-based approach to such an epidemiological study is advantageous as children enrolled on clinical trials are a biased subgroup of all paediatric cancer cases and in particular, will typically exclude patients who die before treatment initiation or those deemed 'too sick' for enrollment. Thus, our objective was to describe the proportion of deaths due to TRM and to identify risk factors and causes of TRM in a population-based cohort.

\section{MATERIALS AND METHODS}

This study was approved by the Research Ethics Board at The Hospital for Sick Children (SickKids), Toronto, Canada and all participating centers. The requirement for informed consent was waived at all sites given the retrospective nature of the study.

The classification system defines TRM as deaths occurring in the absence of progressive cancer (Alexander et al, 2015). For TRM cases, causes of death are classified by evaluating the medical records 2 weeks prior to the death and categories for cause of death are: infection, haemorrhage, thrombosis, cardiac system, immune mediated, metabolic, nervous system, respiratory system, gastrointestinal (GI) system, renal system and external causes. These etiologies are classified as probably, possibly or not the cause of death as previously described (Alexander et al, 2015). The approach allows multiple probable causes of death to be described. It also allows TRM to be designated before starting cancer therapy as treatment initiation in a critically ill child will vary depending on physician and family preferences, and because some of the deaths may be preventable with enhanced supportive care.

Setting and patients. The population consisted of children diagnosed and treated in the province of Ontario, Canada. There are five centers in Ontario that provide care for paediatric cancer patients, namely London Health Sciences Centre (London), Hamilton Health Sciences Centre (Hamilton), SickKids (Toronto), Cancer Centre of Southeastern Ontario at Kingston (Kingston) and Children's Hospital of Eastern Ontario (Ottawa). All centers report the data to the Pediatric Oncology Group of Ontario Networked Information System (POGONIS). POGONIS has been collecting information prospectively on all cases of paediatric cancer diagnosed and treated in the province of Ontario since 1985.

We included all deaths reported to POGONIS between 1 January 2003 and 31 December 2012 and eligible children were $\leqslant 18$ years at cancer diagnosis. We excluded patients that had an unconfirmed cancer diagnosis. Children who had not been seen

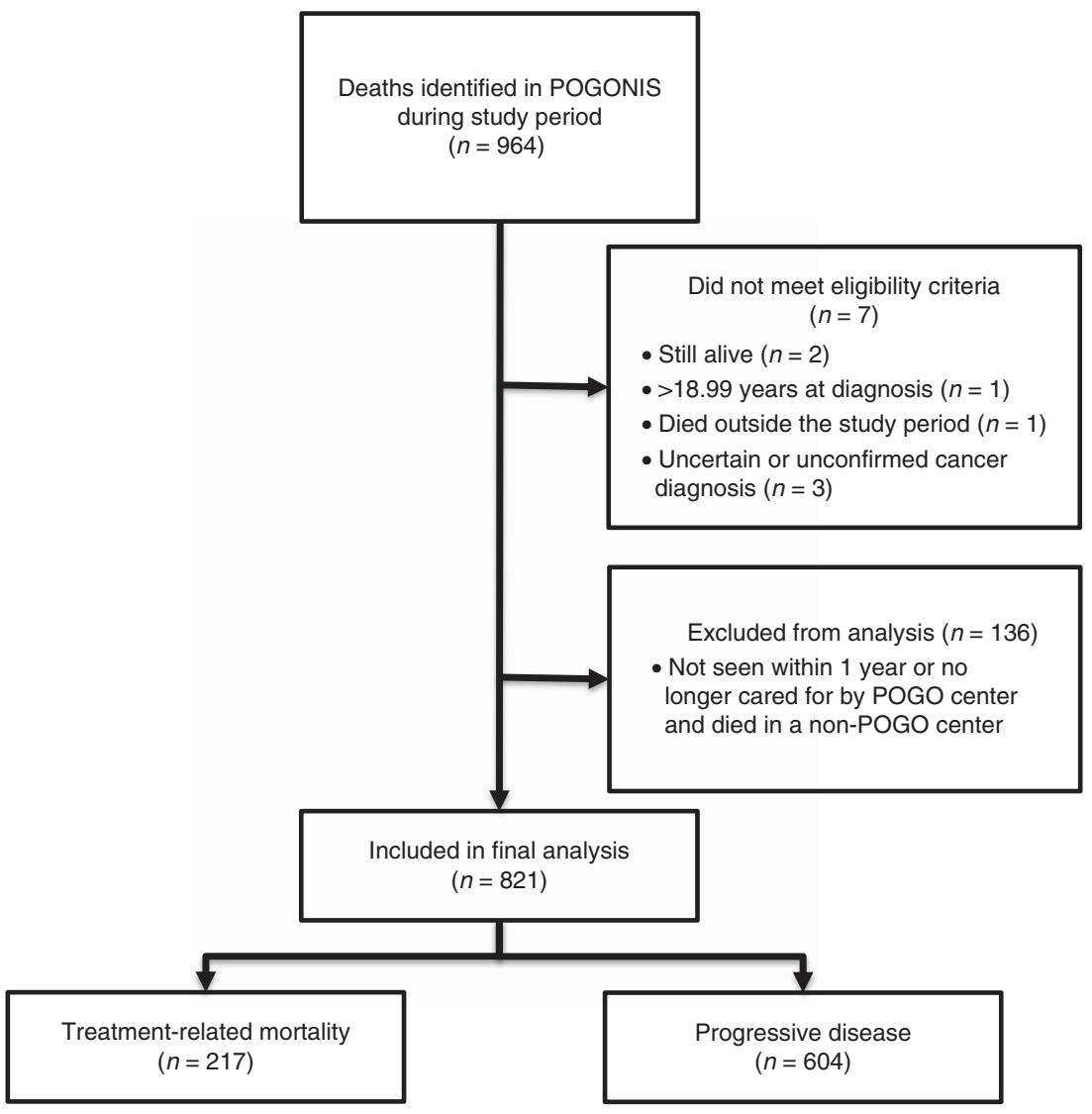

Figure 1. Flow diagram of case identification and selection. 
within 1 year prior to death or who were no longer cared for by clinicians from a Pediatric Oncology Group of Ontario (POGO) center and who died in a non-POGO center were considered inevaluable and excluded from the analysis unless sufficient documentation existed to support a TRM designation.

Design. Trained clinical research associates (CRAs) traveled to each of the five Ontario institutions in order to abstract the required information for TRM designation and cause-of-death attribution. In the designation of TRM, we did not censor at relapse or HSCT as our intent was to describe TRM in all children with cancer. Demographic information was abstracted from the medical records and from the data submitted to POGONIS. The data included details related to diagnosis, relapse, subsequent malignancy and receipt of allogeneic or autologous HSCT. The data regarding receipt of chemotherapy, radiotherapy and cancerremoving surgery were collected within 2 months prior to death.

A group of 6 CRAs received training on the TRM and cause-ofdeath attribution system and their skills were evaluated with 10 randomly chosen cases. Skill level was considered adequate if assignment of TRM was correct in at least 9 of 10 cases; the correct response was determined by two paediatric oncologists (SA and LS). Once competency was deemed adequate, CRAs designated all cases as TRM or not TRM and if TRM, determined the probable and possible causes of death.

Statistics. We described the characteristics of the population and TRM cases using descriptive statistics. Factors associated with TRM were evaluated using univariate and multiple logistic regression, and associations were described using odds ratios (ORs) with 95\% confidence intervals (CIs). Variables considered in the multiple regression model were baseline characteristics and not treatment factors (since these may be in the causal pathway) or factors occurring at the time of death such as withdrawal of supportive care or performing an autopsy (since these would not have been related to the etiology of death). Statistical significance was defined as $P$-value $<0.05$. Statistical analysis was conducted using the SAS statistical program (SAS-PC, version 9.4; SAS Institute Inc, Cary, NC, USA).

Role of the funding source. The study funding source (POGO) had no role in study design, data collection, analysis, interpretation, writing of the report, or decision to submit the paper for publication.

\section{RESULTS}

Among the training cases, TRM designations were correct in 10 out of 10 cases for all 6 CRAs, meeting criteria for adequate skill level. Cause-of-death attributions were completely correct in 10 out of 10 cases for 3 CRAs, 9 out of 10 cases for 2 CRAs and 8 out of 10 for 1 CRA.

There were 964 deaths identified in POGONIS during the study period. Figure 1 illustrates the flow diagram of subject identification and selection and reasons for exclusion. Characteristics of the 821 included cases are shown as Table 1, both for the entire cohort and stratified by underlying diagnosis. The median age at diagnosis

Table 1. Demographics of the study population stratified by underlying diagnosis

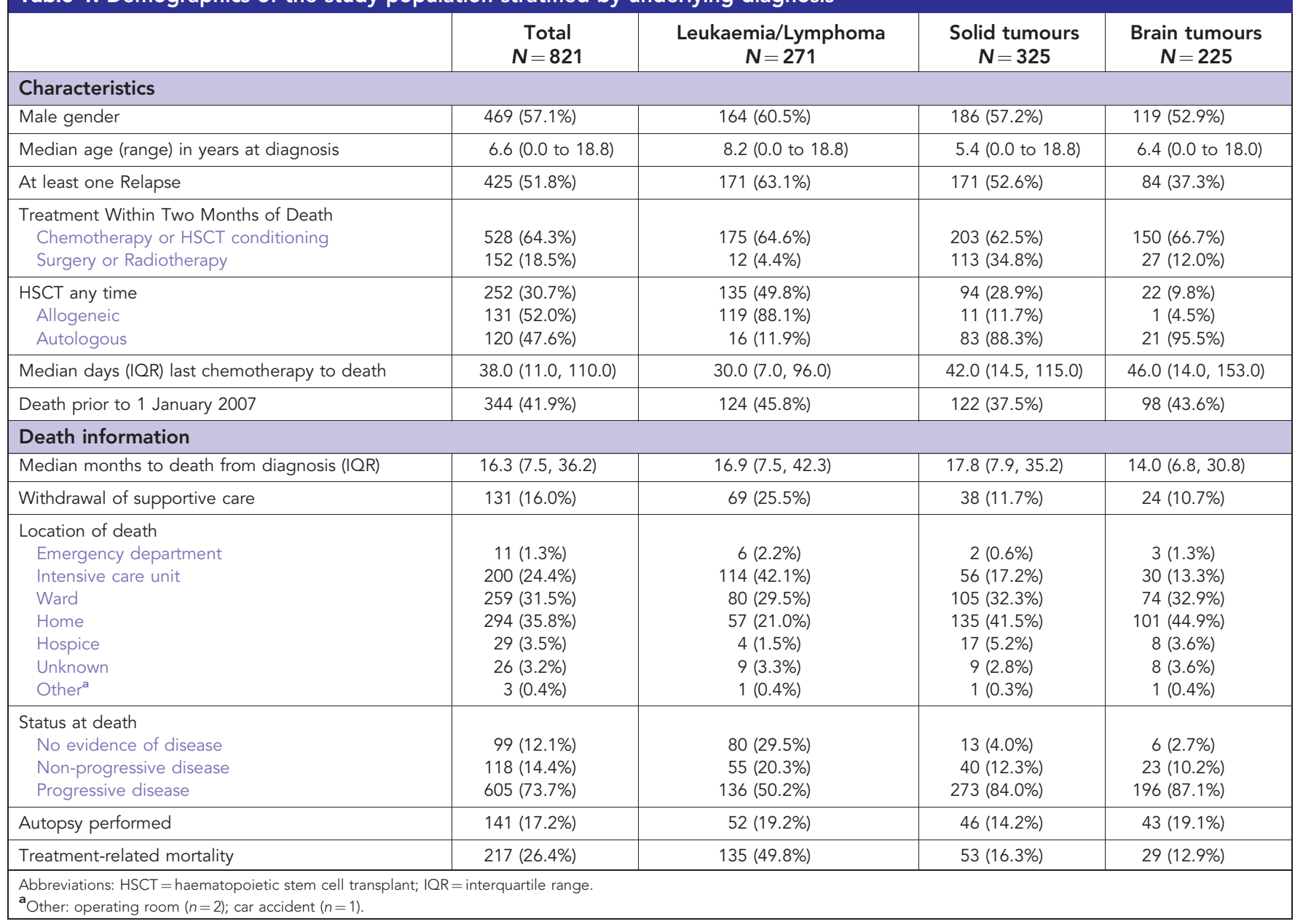


Table 2. Univariate logistic regression evaluating factors associated with Treatment-related mortality

\begin{tabular}{|c|c|c|c|c|}
\hline & $\begin{array}{c}\text { TRM } \\
N=217\end{array}$ & $\begin{array}{l}\text { Progressive disease } \\
\qquad(N=604)\end{array}$ & OR $(95 \% \mathrm{Cl})$ & $P$-value ${ }^{a}$ \\
\hline \multicolumn{5}{|l|}{ Characteristics } \\
\hline Male Gender & 117 (53.9\%) & $352(58.3 \%)$ & $0.84(0.62$ to 1.15$)$ & 0.277 \\
\hline Median Age (Range) in Years at Diagnosis & 5.5 (1.4 to 13.0$)$ & $7.1(0.0$ to 18.8$)$ & 0.96 (0.94 to 0.99$)$ & 0.013 \\
\hline $\begin{array}{l}\text { Underlying Diagnosis } \\
\text { Leukaemia or lymphoma } \\
\text { Solid tumour } \\
\text { Brain tumour }\end{array}$ & $\begin{array}{r}135(62.2 \%) \\
53(24.4 \%) \\
29(13.4 \%)\end{array}$ & $\begin{array}{l}136(22.5 \%) \\
272(45.0 \%) \\
196(32.5 \%)\end{array}$ & $\begin{array}{c}5.11(3.52 \text { to } 7.51) \\
\text { REF } \\
0.76(0.46 \text { to } 1.23)\end{array}$ & $<0.0001$ \\
\hline Relapse & $66(30.4 \%)$ & 359 (59.4\%) & $0.30(0.21$ to 0.41$)$ & $<0.0001$ \\
\hline $\begin{array}{l}\text { Treatment within two months of death } \\
\text { Chemotherapy } \\
\text { Surgery or radiotherapy }\end{array}$ & $\begin{array}{c}163(75.1 \%) \\
20(9.2 \%)\end{array}$ & $\begin{array}{l}365(60.4 \%) \\
132(21.9 \%)\end{array}$ & $\begin{array}{l}1.98(1.40 \text { to } 2.82) \\
0.36(0.21 \text { to } 0.58)\end{array}$ & $\begin{array}{r}0.0001 \\
<0.0001\end{array}$ \\
\hline $\begin{array}{l}\text { HSCT any time } \\
\text { Allogeneic } \\
\text { Autologous }\end{array}$ & $\begin{array}{l}81(37.3 \%) \\
68(84.0 \%) \\
13(16.0 \%)\end{array}$ & $\begin{array}{r}170(28.1 \%) \\
63(37.1 \%) \\
107(62.9 \%)\end{array}$ & 1.54 (1.11 to 2.14$)$ & 0.009 \\
\hline \multicolumn{5}{|l|}{ Death information } \\
\hline Median months to death from diagnosis (IQR) & $7.8(0.9$ to 25.4$)$ & $18.3(10.2$ to 38.1$)$ & 0.99 (0.99 to 1.0$)$ & 0.019 \\
\hline Withdrawal of supportive care & $100(46.1 \%)$ & $31(5.1 \%)$ & 15.77 (10.18 to 25.05$)$ & $<0.0001$ \\
\hline $\begin{array}{l}\text { Location of death } \\
\text { Emergency department } \\
\text { Intensive care unit } \\
\text { Ward } \\
\text { Home } \\
\text { Hospice } \\
\text { Unknown } \\
\text { Other }\end{array}$ & $\begin{aligned} 5 & (2.3 \%) \\
162 & (74.7 \%) \\
36 & (16.6 \%) \\
8 & (3.7 \%) \\
& 2(0.9 \%) \\
1 & (0.5 \%) \\
3 & (1.4 \%)\end{aligned}$ & $\begin{aligned} 6 & (1.0 \%) \\
38 & (6.3 \%) \\
223 & (36.9 \%) \\
285 & (47.2 \%) \\
27 & (4.5 \%) \\
25 & (4.1 \%) \\
0 & (0.0 \%)\end{aligned}$ & & $<0.0001$ \\
\hline Autopsy Performed & $87(40.1 \%)$ & 54 (8.9\%) & $6.82(4.63$ to 10.11$)$ & $<0.0001$ \\
\hline
\end{tabular}

was 6.6 years (range $0-18.8$ ) and $51.8 \%$ of subjects had at least one relapse. Overall, $30.7 \%$ had undergone HSCT with a similar number receiving allogeneic and autologous transplants although leukaemia and lymphoma patients predominantly had allogeneic HSCTs while patients with solid and brain tumours predominantly had autologous HSCTs. The median number of months from diagnosis to death was 16.3 (interquartile range 7.5-36.2). For all children combined, TRM occurred in 217/821 (26.4\%) of deaths. Seventeen of the TRM cases died before cancer treatment was received.

Table 2 identifies factors associated with TRM. There was no association between TRM and more recent death (on or after 1 January 2007). The following were significantly positively associated with TRM in univariate analysis: leukaemia/lymphoma vs solid tumour, absence of relapse, chemotherapy or absence of surgery/radiotherapy within 2 months of death, receipt of HSCT, shorter time from diagnosis to death, withdrawal of supportive care, location of death and performing an autopsy. In multiple regression which considered underlying diagnosis, age and relapse, all three factors were independently associated with TRM. Increasing age at diagnosis (OR: 0.92, 95\% CI: 0.89-0.95), brain tumour (OR: 0.54, 95\% CI: 0.32-0.91) and relapse (OR: 0.11, 95\% CI: $0.07-0.17)$ were associated with a lower risk of TRM, while leukaemia/lymphoma (OR: 11.18, 95\% CI: 6.94-18.60) was associated with a higher risk of TRM.

Most TRM cases had multiple probable or possible causes of death with only $34(15.7 \%)$ having a single probable or possible cause of death. Probable causes of death are shown in Table 3. The most common probable causes of death were respiratory system failure followed by infection, haemorrhage, GI system failure and nervous system failure. Most of the 140 deaths with respiratory system failure as a probable cause of death were concurrent with infection as another cause of death. More specifically, 87 (62.1\%) cases of respiratory system failure as a probable cause of death were concurrent with infection as a probable cause of death and 31 (22.1\%) were concurrent with infection as a possible cause of death. Among the 17 who died before cancer treatment initiation, the most common probable causes of death were nervous system failure $(n=10)$, respiratory system failure $(n=7)$, haemorrhage $(n=8)$ and infection $(n=4)$.

Among TRM cases, the proportion of patients with probable causes of death for the following etiologies differed based on underlying diagnosis: respiratory system failure, infection, GI system failure, nervous system failure and renal system failure. More specifically, respiratory system failure as a probable cause of death was most common among solid tumour patients, while infection as a probable cause of death was most common among leukaemia/lymphoma patients and least common among brain tumour patients. GI system failure as a probable cause of death was most common among solid tumour patients while nervous system failure as a probable cause of death was most common among brain tumour patients. Thrombosis, metabolic and external causes were rare causes of death.

Table 4 and Supplementary Material explore infectious probable causes of TRM. Bacteria were more common than fungi and the most common infections associated with TRM were coagulase negative Staphylococcus $(n=19)$, Aspergillus species $(n=10)$ and Candida spp $(n=17)$. Fungal infections were most common 
Table 3. Probable Causes of Treatment-Related Mortality Stratified by Underlying Diagnosis

\begin{tabular}{|c|c|c|c|c|c|}
\hline & $\begin{array}{c}\text { Total } \\
N=217\end{array}$ & $\begin{array}{l}\text { Leukaemia/Lymphoma } \\
\qquad N=135\end{array}$ & $\begin{array}{l}\text { Solid tumours } \\
\qquad N=53\end{array}$ & $\begin{array}{l}\text { Brain tumours } \\
\qquad N=29\end{array}$ & $P$-value ${ }^{a}$ \\
\hline \multicolumn{6}{|c|}{ Probable cause of death } \\
\hline Respiratory System & $140(64.5 \%)$ & 87 (64.4\%) & $43(81.1 \%)$ & $10(34.5 \%)$ & 0.0001 \\
\hline Infection & 109 (50.2\%) & 78 (57.8\%) & $26(49.1 \%)$ & 5 (17.2\%) & 0.0004 \\
\hline Haemorrhage & 80 (36.9\%) & 47 (34.8\%) & 21 (39.6\%) & 12 (41.4\%) & 0.715 \\
\hline GI System & $64(29.5 \%)$ & $38(28.1 \%)$ & 23 (43.4\%) & $3(10.3 \%)$ & 0.006 \\
\hline Nervous System & $63(29.0 \%)$ & 35 (25.9\%) & $12(22.6 \%)$ & $16(55.2 \%)$ & 0.004 \\
\hline Renal System & $59(27.2 \%)$ & 42 (31.1\%) & $16(30.2 \%)$ & 1 (3.4\%) & 0.009 \\
\hline Cardiac & 38 (17.5\%) & $23(17.0 \%)$ & $13(24.5 \%)$ & 2 (6.9\%) & 0.129 \\
\hline Immune Mediated & $28(12.9 \%)$ & $19(14.1 \%)$ & 9 (17.0\%) & $0(0.0 \%)$ & 0.073 \\
\hline Thrombosis & 7 (3.2\%) & $7(5.2 \%)$ & $0(0.0 \%)$ & $0(0.0 \%)$ & 0.111 \\
\hline Metabolic & $4(1.8 \%)$ & $3(2.2 \%)$ & $1(1.9 \%)$ & $0(0.0 \%)$ & 0.722 \\
\hline External Causes & $1(0.5 \%)$ & $1(0.7 \%)$ & $0(0.0 \%)$ & $0(0.0 \%)$ & 0.737 \\
\hline
\end{tabular}

\section{Table 4. Microbiological Causes of Treatment-Related Mortality}

\begin{tabular}{|c|c|c|c|c|}
\hline Organism Name ${ }^{a}$ & $\begin{array}{c}\text { Total } \\
N=109\end{array}$ & $\begin{array}{l}\text { Leukaemia/lymphoma } \\
\qquad N=76\end{array}$ & $\begin{array}{l}\text { Solid tumours } \\
\qquad N=27\end{array}$ & $\begin{array}{c}\text { Brain tumours } \\
\qquad N=6\end{array}$ \\
\hline Gram positive & 45 & 32 & 8 & 5 \\
\hline Coagulase negative Staphylococcus & 17 & 13 & 3 & 1 \\
\hline Enterococcus spp & 5 & 3 & 1 & 1 \\
\hline Staphylococcus aureus & 5 & 4 & 1 & 0 \\
\hline Viridans group streptococci & 4 & 3 & 1 & 0 \\
\hline Streptococcus NOS & 3 & 1 & 1 & 1 \\
\hline Clostridium septicum & 1 & 1 & 0 & 0 \\
\hline Micrococcus NOS & 1 & 1 & 0 & 0 \\
\hline Gram negative & 28 & 17 & 10 & 1 \\
\hline Pseudomonas aeruginosa & 8 & 6 & 2 & 0 \\
\hline Enterobacter cloacae & 5 & 2 & 2 & 1 \\
\hline Escherichia coli & 4 & 2 & 2 & 0 \\
\hline Fungal & 36 & 27 & 9 & 0 \\
\hline Aspergillus spp & 10 & 8 & 2 & 0 \\
\hline Candida non-albicans & 9 & 8 & 1 & 0 \\
\hline Candida albicans & 7 & 3 & 4 & 0 \\
\hline Fungi NOS & 4 & 3 & 1 & 0 \\
\hline Alternaria NOS & 1 & 1 & 0 & 0 \\
\hline Candida NOS & 1 & 1 & 0 & 0 \\
\hline Fusarium solani & 1 & 1 & 0 & 0 \\
\hline Mucormycosis & 1 & 1 & 0 & 0 \\
\hline Mold NOS & 1 & 0 & 1 & 0 \\
\hline Pneumosystis jirovecii & 1 & 1 & 0 & 0 \\
\hline
\end{tabular}

among those with leukaemia or lymphoma. The most common sites of infection for TRM cases were blood $(n=112)$ and tissue biopsy $(n=23)$.

\section{DISCUSSION}

Using the newly developed international consensus definitions of TRM, approximately one in four paediatric cancer deaths were classified as TRM. Independent risk factors for TRM were younger age, underlying diagnosis and absence of relapse. The most common probable causes of TRM were respiratory system failure, infection and haemorrhage, and probable causes of TRM differed by underlying diagnosis. This study is important as paediatric patients receiving intensive treatments still experience unacceptably high TRM (Balduzzi et al, 2002; Creutzig et al, 2004). Our results are foundational as they provide a basis for future epidemiological studies of TRM and design of supportive care strategies to reduce toxic mortality.

We found that the proportion of deaths due to TRM did not change over time. In contrast, others have noted reduced TRM over time when intensive therapies are introduced, such as for acute myeloid leukaemia treatment and HSCT (Jastaniah et al, 
2012; Altshuler et al, 2016). One explanation for this discrepancy is the different metric used, as we evaluated the proportion of deaths as opposed to the proportion of patients with TRM. An important next step would be to evaluate a cohort of newly diagnosed paediatric cancer patients and to describe the TRM rate and risk factors for TRM among these patients.

We also found that leukaemia diagnosis, younger age and absence of relapse were risk factors for TRM. Most studies of TRM have focused on specific patient populations and thus, our approach is unique. The finding of increased TRM in patients with leukaemia/lymphoma and patients without relapse is expected. However, the finding of increased TRM in younger patients is surprising, given that in the context of acute myeloid leukaemia, older age (Canner et al, 2013) and obesity (Lange et al, 2005) have been associated with increased TRM. These conflicting data may relate to the limited upper age range in our study (18 years) or the difference in metric as previously described. We also found that invasive fungal disease, and in particular, both molds and yeasts are common probable causes of TRM. This finding suggests that approaches to prevent and treat fungal disease should be a priority for future research.

We previously asked whether CRAs can abstract this type of data or whether physicians should ultimately be classifying TRM and cause-of-death attributions. We found that with minimal training, all CRAs were able to correctly distinguish TRM vs progressive cancer and that attribution classification was excellent. We will continue to develop educational materials to enhance reliable TRM and cause-of-death attribution for future studies.

The strength of this study is the use of consistent and trained CRAs to abstract all the data for this analysis. A second strength is the population-based nature of the study, which improves generalizability and avoids selection bias. However, our results must be interpreted in light of its limitations. The most important limitation is that, we did not describe the TRM rate among all newly diagnosed cancer patients. We took the approach of first evaluating TRM among all deaths as a pragmatic initial step since we needed to evaluate all deaths to determine which ones were TRM and evaluation of TRM among all cancer patients will require the additional data about those who did not die. Nonetheless, evaluating the TRM rate among newly diagnosed cancer patients is planned as a future next step. Also, we often did not have adequate documentation to classify TRM for patients who transitioned to adult care, a subgroup of patients of particular importance given known disparities for adolescent and young adult patients (Nachman, 2003).

In conclusion, TRM was responsible for $26.4 \%$ of deaths in paediatric cancer. Underlying diagnosis, younger age and absence of relapse were associated with TRM and causes of TRM differed by diagnosis group. Future work should evaluate TRM rate and risk factors among newly diagnosed cancer patients.

\section{ACKNOWLEDGEMENTS}

This work was supported by an Operating Grant from the Pediatric Oncology Group of Ontario. LS had full access to all the data in the study and had final responsibility for the decision to submit for publication. We thank Michelle Lee and Biljana Gillmeister who conducted early TRM designations.

\section{CONFLICT OF INTEREST}

The authors declare no conflict of interest.

\section{REFERENCES}

Alexander S, Pole JD, Gibson P, Lee M, Hesser T, Chi SN, Dvorak CC, Fisher B, Hasle H, Kanerva J, Moricke A, Phillips B, Raetz E, Rodriguez-Galindo C, Samarasinghe S, Schmiegelow K, Tissing W, Lehrnbecher T, Sung L, International Pediatric Oncology Mortality Classification G (2015) Classification of treatment-related mortality in children with cancer: a systematic assessment. Lancet Oncol 16(16): e604-e610.

Altshuler C, Haley K, Dhall G, Vasquez L, Gardner SL, Stanek J, Finlay JL (2016) Decreased morbidity and mortality of autologous hematopoietic transplants for children with malignant central nervous system tumors: the 'Head Start' trials, 1991-2009. Bone Marrow Transplant 51(7): 945-948.

Balduzzi A, Valsecchi MG, Silvestri D, Locatelli F, Manfredini L, Busca A, Iori AP, Messina C, Prete A, Andolina M, Porta F, Favre C, Ceppi S, Giorgiani G, Lanino E, Rovelli A, Fagioli F, De Fusco C, Rondelli R, Uderzo C, Associazione Italiana Ematologia Oncologia Pediatrica BMTG (2002) Transplant-related toxicity and mortality: an AIEOP prospective study in 636 paediatric patients transplanted for acute leukemia. Bone Marrow Transplant 29(2): 93-100.

Blanco E, Beyene J, Maloney AM, Almeida R, Ethier MC, Winick N, Alexander S, Sung L (2012) Non-relapse mortality in paediatric acute lymphoblastic leukaemia: a systematic review and meta-analysis. Leuk Lymphoma 53(5): 878-885.

Canner J, Alonzo TA, Franklin J, Freyer DR, Gamis A, Gerbing RB, Lange BJ, Meshinchi S, Woods WG, Perentesis J, Horan J (2013) Differences in outcomes of newly diagnosed acute myeloid leukaemia for adolescent/ young adult and younger patients: a report from the Children's Oncology Group. Cancer 119(23): 4162-4169.

Craft AW (2000) Childhood cancer-mainly curable so where next? Acta Paediatr 89(4): 386-392.

Creutzig U, Zimmermann M, Reinhardt D, Dworzak M, Stary J, Lehrnbecher T (2004) Early deaths and treatment-related mortality in children undergoing therapy for acute myeloid leukaemia: analysis of the multicenter clinical trials AML-BFM 93 and AML-BFM 98. J Clin Oncol 22(21): 4384-4393.

Ethier MC, Blanco E, Lehrnbecher T, Sung L (2011) Lack of clarity in the definition of treatment-related mortality: paediatric acute leukaemia and adult acute promyelocytic leukaemia as examples. Blood 118(19): 5080-5083.

Jastaniah W, Abrar MB, Khattab TM (2012) Improved outcome in paediatric AML due to augmented supportive care. Pediatr Blood Cancer 59(5): 919-921.

Lange BJ, Gerbing RB, Feusner J, Skolnik J, Sacks N, Smith FO, Alonzo TA (2005) Mortality in overweight and underweight children with acute myeloid leukaemia. JAMA 293(2): 203-211.

Nachman JB (2003) Adolescents with acute lymphoblastic leukaemia: a new 'age'. Rev Clin Exp Hematol 7(3): 261-269.

Tran TH, Lee M, Alexander S, Gibson P, Bartels U, Johnston DL, Portwine C, Silva M, Pole JD, Sung L (2014) Lack of treatment-related mortality definitions in clinical trials of children, adolescents and young adults with lymphomas, solid tumors and brain tumors: a systematic review. BMC Cancer 14: 612.

This work is published under the standard license to publish agreement. After 12 months the work will become freely available and the license terms will switch to a Creative Commons AttributionNonCommercial-Share Alike 4.0 Unported License.

Supplementary Information accompanies this paper on British Journal of Cancer website (http://www.nature.com/bjc) 